Um den transplantierten Nasenflügel die symmetrische, feine Form der anderen Seite geben zu können, machte ich noch dreimal, zuletzt am 20. Februar 1916, kleinere Eingriffe - mit kosmetisch ausgezeichnetem Erfolg. Dabei ist an Ohrdefekt kaum etwas zu bemerken. Patientin hat ihre Haartracht nach ihrer Heilung so gestaltet, daß sie ihr zur Plastik benutztes kleineres und grazileres Ohr frei ließ und das andere unberührte $\mathrm{Ohr}$ teilweise mit ihrer Haartracht bedeckte.

Ich kann den Effekt der Operat:on, nachdem nun über $2^{1 / 2}$ Jahre verflossen sind, als definitiv und vollständig befriedigend bezeichnen.

Das transplantierte Ohrmuschelstiick hat auf meine Bitte Prof. Julius Donath bezüglich der Nervenfunktion am 7. Mai 1917, also 26 Monate nach der Operation, untersucht 1nd hat uns folgenden Befund zur Verfügung gestellt: „Die vordere Hälfte des rechten Nasenflügels wurde mit einem Baumwollfaden geprüft. Bei den wiederholten Prüfungen wird derselbe meist prompt signalisiert.

Vom Sievekingschen Aesthesiometer werden die beiden Spitzen

Aus der 11. Chirurgischen Abteilung des hauptstädtischen Krankenhauses St. Rochus in Budapest.

\section{Partielle Nasenplastik mit freier Transplantation aus der Ohrmuschel.}

\section{Von J. P. Haberern, Vorstand der Abteilung.}

Teile der Ohrmuschel wirden seit Fritz Königs Mitteilung, Fe bruar 1902, mehrfach als Ersatz von Nasendefekten durch freie Transplantation benutzt. Dies Verfahren hat unter andern die außerordentlich günstige kosmetische Bedeutung, daß dabei das Gesicht des Patienten durch kleinere oder größere Narben nicht verunstaltet wird, wie das bei anderen Methoden an den benachbarten Teilen der Nase, des Gesichts, der Stirne leider sehr häufig der Fall ist. Darum ist es wichtig, daß die plastische Chirurgie diese Methode an!gedehnter benutze und vervollkommne. Es ist dies eine sehr dankbare Aufgabe, anch jetzt recht zeitge mä $B$, da diese Methode auch in der Kriegschirurgie häufig Verwe nd ung finden kann.

Frau M. D., 18 Jahre alt, wurde am 16. Februar 1915 auf meine Chirurgische Abteilung aufgenommen. Sie ist am 15. Februar angeblich mit dem Messer in der Hand gestürzt, wobei sie sich die Nase schwer verletzte. Bei der sonst gesunden Frau fehlt ungefähr die Hälfte des rechten Nasenflügelr, und zwar gegen die Nasenspitze zu. Der Defekt ist, dem Rande des Nasenflügels entsprechend, $20 \mathrm{~mm}$ lang. Die Höhe des Defektes gegen die Nasenwurzel $\mathrm{zu}$ ist $13 \mathrm{~mm}$. Gegen die Nasenspitze zu ist eine breite, schiefe Narbe sichtbar, nach hinten ist die Narbenfläche steil, glatt.

Opsration am 13. März am Ohre, wie an der Nase in lokaler Anästhesie. Nach Auffrischung der Defektränder wurde aus dem oberen Teil der Ohrmuschel ein der Form des Defektes entsprechendes Stück aurgeschnitten und durch freie Transplantation in den Defekt vernäht, und zwar so, daß das knorpelige, innen und außen mit Haut bedeckte Ohrmuschelstück mit äußerer Haut- und innerer Haut-Schleim háa tnaht fixiert wurde. Das Nasenloch wurde mit steriler Gaze leicht tamponicrt und der Tam. pon zweimal täglich gewechselt.

Nach der Operation wurde das transplantierte Stück etwas bläu. lich verfärbt, etwas ödematös und kalt. Es wurde nicht bullös, ez zoigte sich nur eine leichte seröse Sekretion an der Stelle der Naht, wodurch eine oberflächliche, zarte Kruste entstand. Am dritten Tage nachhcr war das implantierte Stück etwas wärmer anzufühlen, die aschblaue Farbe wurde um eine Spur heller, etwas rötlich, bis es am fünften Tage kirschrote Färbung zeigte. In ganz kirzer Zeit nahm es eine normalo Färbung an, die sich von den Nachbarpartien bezüglich der Färbung nicht mehr unterschied. Die Nähte wurden zum Teil am achten Tage entfernt, die letzten zehn Tage nach der Operation, am 23. März Heilung mit glatter Narbe prima intentione.

Am 9. August wurde sie wieder aufgenommen, um die kleinen nachträglichen plastischen' Korrekturen vorzunehmen.

An der Nasenspitze legte sich das transplantierte Stück, mit nicht ganz feinen Konturen an, und so mußte es nach der Form des anderen Nasenloches geformt werden. Dies gelang schön durch das Ausschneiden eines kleinen Keiles aus dem vorspringenden Teile an der Nasenspitze. Natürlich führte ich den Schnitt bis zum Knorpel am inneren Teile der Nasenöffnung, dann präparierte ich das Haut tück auf, entfernte aus dem Knorpel das entsprechende keilförmige Stück und nähte die aufpräparierte Haut an der Innenfläche wieder zurück.

Nach diesem kleinen Eingriff wurde das den Nasenflügel bildende, transplantierte Ohrmuschelstück etwas kühler und etwas bläulich verfärbt - trotzdem ich bei der Operation jede derbe Berührung oder Druck vermied, mit den feinsten Instrumenten und zarter Berührung der Gewebe vorging. Den nächsten Tag erholte sich alles vollständig. Entfernung der Nähte am sechsten Tage. Der an das Septum grenzends Teil des Nasenflügels wurde nun vollständig gleich mit der Ecke der anderen Nazenflügelscite. noch in $2 \mathrm{~mm}$ Entfernung angegeben, wie auch auf der entsprechenden Stelle der gesunden Seite, mit dem Unterschiede, daß die beiden Spitzen auf dem Transplantate stumpfer gefühlt werden. In $1 \mathrm{~mm}$ Entfernung wird sowohl auf dem Transplantat; wie auf der gesunden Seite nur eine Spitze empfunden. Auch die Wärme- und Kälteempfindung ist prompt auf dem Transplantat, wenngleich herabgesetzt.

Das Transplantat zejgt demnach entschiedene, wenngleich herabgesetzte Tast-, Schmerz-, Kälte- und Wärmeempfindung. Dasselbe ist nicht nur, wie die Färbung zeigt, vaşkularisiert, sondern es ist auch die Nervenleitung wieder hergestellt. Für letztere mu $B$ als besonders beweisend erachtet wcrden die Schmerz- und Temperaturempfindung. Eine direkte Neubildung von Gefäßkapillaren und Nervenfasern ist mindestens in begrenzendem Narbergewebe anzunehmen." "

König weist auf die Häufigkeit der Nazenflügeldefekte hin. Er sah Defekte der Nazenflügel am häufigsten durch Lupus, durch tuberkulöse Prozesse und durch Thermokauter entstehen, diet durch die ganze Dicke des Nasenflügels gehen. Am Nasenflügel sind selbst kleine Defekte entstollend. Diese Operation hat König auf Grund der Erfahrung versucht, daß selbst große, ganz abgeschlagene Stücke der Ohrmuschel bei guter Adaptation wieder anheilen, und ebenso kann auch ein $\mathrm{Ohr}$ muschelstück an dem mit Blutgefäßen sehr gut versorgten Nasenflïgel anhoilon. Dis Haut der Ohrmuschel, beinahe ohne Unterhautfettgewebe, eignot sich vorzüglich zur Verpflanzung als ungestielter Lappen. Allbekannt, $a ß 3$ Krorpel sich gut verpflanzen läßt und an der neuen Stelle sich erhält. (v. Mangold..) Knorpel hat keine Blutgefäße und kann bei goringen Ernährungsverhältnissen weiter leben, wichtig ist nur eine genaue Adaptation und das Anlegen der fixierenden Nähte ohne Druck und Spannung. Endlich meint König, daß die Transplantation eines Ohrmuschelstiickes in den Najenfliigeldefekt die am meist physiologische Form der Pla tik ist, da in allen drei Schichten analoges Mater: al vcrpflanzt wird.

Hier wollen wir erwähnen, daß Büdinger Ohrmuschelteile bei augenärztlichen Opcrationen zur Anwenaung brachte, wio er dies in einer Juni 1912 erschienenen Arbeit, al o vicr Monate nach Königs Mitteilung, veröffentlichte. Das Wesen des Büdingerschen Verfahrens ist, daß er den entfernton tarealon Teil des Augenlides durch den Hautknorpollapnen der Ohrmuschel ersetzt. Den Defekt des Ohres vereinigte er mit Nähten, nachdem er die Deformation durch Entfernung eines Keiles aus dem Rande der Ohrmuschel ausgrglichen.

Birch-Hirschfeld faßt die Indikationen der Büdingerschen Tar opla tik in Folgendem zusammen. Es eignen sich hierzu Augenlidtumorer, wie sie sich entweder aus der Bindehaut des Tarsus entwickeln oder sekundär auf den Tarsus übergreifen, vorausgesetzt, daß in diesen Fällen keine größere, mit der Entfernung des Bulbus einhergehende Operation nöt $g$ ist.

Gute Dienste kann dieses Verfahren auch in jenen Fällen von Trachom und Tuberkulose leisten, bei welchen auch der Tarsus in Mitleidenschaft gezogen ist, ebenso bei Augenlidkolobom und Verletzungen, die den teilweisen oder vollständigen Ercatz des Tarsus benötigen.

- Auch nach den anatomischen Untersuchungen Birch-Hirschfelds erscheint der Ohrknorpel mit seiner Hautbedeckung zum Ersatz des tarcalen Teiles des Augenlides geeignet, weil die äünne Knorpelschicht durch eine schmale Lage dichten Bindegewebes mit dem mehrschichtigen Epithel verbunden ist, welche Epithelzellen sehr ähnlich sind, mit den Epithelzellen der tarcalen Bindehaut. Haare, Drüsen und andere Unebenheiten fehlen fa:t gänzlich.

Transplantationsversuche haben bewiesen, daß der Knorpel bezüglich der Ernährung am Mutterboden relativ geringe Ansprüche macht und daher leichter einheilt als andere Gewebsarten.

Birch-Hirschfèld überzeugte sich mikroskopisch, daß zwei bis drei Wochen nach solchen Transplantationen am Kaninchen der Knorpel in den normalen Tarsus einheilte und vom normalen Tarsus durch eine schmale, bindegewebige Zone begrenzt war. Die Form und Färbbarkeit des Knorpsls und des Epithols blicben normal. In einem Falle, bri c.cm 
eine Infektion zustande kam und die Lider zwei Tage nach der Operation anschwollen und sich schleimig-eitrige Sekretion einstellte, stieß sich ein größeres Gewebsstück ab, das für das transplantierte Knorpelstück gehalten wurde. Zwölf Tage nach der Operation schwand die Sekretion, und es zeigte sich, daß der Knorpel im Niveau des Defektes glatt erhalten war, keine Nekrose, nur an der Oberfläche in der Mitte vom Epithel entblc̈Bt war. Das Epithel der Umgebung aber zog sich von den Rändern auf die Knorpeloberfläche, daß also trotz der überstandenen Entzündung das Knorpelstück einheilte. Birch.Hirschfeld meint, daß, selbst wenn sich der Knorpel nach längerer Zeit recorbieren würde, wie nach Implantation in dieLeber, derselbe mit der Zeit durch festes Bindegewebe ersetzt werden würde, was dem Augenlidteil eine größere Festigkeit geben würde, als wir das durch Transplantation durch Haut und Schleimhaut erreichen könnten.

Nach Königs Mitteilung versuchte auch W. Müller bei Lupus die Königsche Methode. Interessant ist in dieser Mitteilung der folgende Fall: 49 jährige Frau, bei der das ganze untere Augenlid wegen Endothcliom entfernt werden mußte (3. Februar 1903). Es wurde ein $3 \frac{1}{2} \mathrm{~cm}$ langes Ohrmuschelstück aus dem rechten Helix in vollem Durchmesser in den Defekt des Augenlides implantiert, so, daß die eine Hautoberfläche des Ohrenstückes mit der Haut des Augenlides, die andere Hautoberfläche mit dem Rest der Bindehaut vereinigt wurde, der zwischenliegende Knorpel ersetzt den Tarsus. Der Lappen ist gut eingeheilt, kosmetischer Effekt ausgezeichnet, weder Ek- noch Entropium, Augenlidschluß vollständig. Nach der Ansicht der Verfasser ist diese Methode des Augenlidersatzes ideal zu nennen, da der funktionelle wie der kosmetische Erfolg vorzüglich ist. Weder das Auge noch die benachbarten Teile zeigen narbige, störende Veränderungen. Der freie Rand der Ohrmuschel gibt einen vorzüglichen Augenlidrand.

Bei Mißlingen der Operation kann das andere Ohr erneuert benutzt werden.

Bei einer 50 jährigen Frau, bei der an beiden unteren Augenlidern nach Lupus Ektropium entstanden war, wurde das rechte untere Augenlid durch gestielten Lappen ersetzt, das linke Augenlid aber durch freie Ohrmuscheltransplantation operiert. Bei der Operation am rechten Augenlid wurde der gestielte Lappen aus der Haut der Backe entnommen und die so entstandene Wunde mit Thiersch-Lappen aus dem rechten Oberarm gedeckt. Der Erfolg war am rechten nicht ganz befriedigend, am linken mit der Ohrmuscheltransplantation kosmetisch und funktionell ausgezeichnet.

Elter und Haas haben in einer späteren Mitteilung erwähnt daß Büdinger zum Ersatz der Bindehaut und des Tarsus einen Teil der Ohrmuschel benutzt hat, die äußere Haut des Augenlides aber mit einem gestielten Lappen aus der Nachbarschaft ersetzte. Indem er neben den freien Lappen auch einen gestielten benutzte, kam eine gewisse Verunstaltung der benachbarten Teile zustande. Der Erfolg der Verfasser ist also kosmetisch besser und auch die Operation einfacher.

Nach Lexer wurde die Königsche Methode der Nasenplastik von mehreren ausgeführt. Das der Ohrmuschel entnommene keilförmige Stück wurde in einigen Fällen nekrotisch. Dies kann die Folge der Wundinfektion sein oder die Folge der geringeren Ernährung, die nur vom Defektrand aus geschehen kann.

Er empfiehlt daher, den Defektrand nicht einfach aufzufrischen, sondern den aufgefrischten Streifen nach innen zu legen, sodaß er mit der Nasenschleimhaut in Verbindung bleibe und dadurch eine breitere Berührungsfläche der Gewebe entstehe.

Weiter meint Lexer, daß diese Nasenflügelplastik den schönsten Erfolg hat, wenn man den partialen Nasenflügeldefekt vergrößert und den ganzen Nasenflügel bis zu seinen normalen Grenzen und Vertiefungen ersetzt. Dadurch erhält er immer gut genährtes Material, und überflüssige Gewebsreste können zur Unterfütterung verwendet werden. Solch ein Nasenflügel wird durch Narben nicht entstellt. Nur wenn diese Narben den normalen Konturen entsprechen, ist der kosmetische Effekt - seiner Meinung nach - vollständig. Ich glaube, dieser Le xersche Vorschlag kann sich nicht auf solche Fälle beziehen, wie der von uns mitgeteilte, wo es sich nicht um Ersatz durch pathologische Prozesse entstandener Defekte handelt, sondern durch Trauma entstandene, glattrandige Defekte vorhanden sind, bei denen sich nicht breite Narben bündel in die Peripherie erstrecken. Bei solchen traumatischen Defekten wäre es vielleicht überflüssig, den ganzen Nasenflügel vor der Operation zu entfernen, nachdem in solchen Fällen die Narben an den vollständig gesunden benachbarten Teilen mit der Ohrmuschel kaum sichtbare Narben bilden, wie wir dies auch in unserem Falle sahen.

Es ist vielleicht von gutem Nutzen, wenn ich er'wähne, daß in einem Lexerschen Falle, wo er die abgebissene Nasenspitze durch ein ovales Ohrmuschelstück ersetzte, der unruhige Patient dieses in der Nacht abgerieben hatte. Er empfiehlt daher, in solchen Fällen eine gewisse Zeit hindurch zum Schutze der Nase die Klappsche Glassaugglocke mit Heftpflasterstreifen an das Gesicht zu fixieren.

Ich wiederhole, daß wirzum Ersatz der Nasenflügel oder Nasen- spitze die Ohrmuschel benu tzen sollen, da dies ein ausgezeichnetes physiologisches Material abgibt, wobei das Ohr kometirch nicht leidet und das Gesicht vollständig von Narben frei bleibt.

$\mathrm{Daß}$ das $\mathrm{Ohr}$ nach vollständiger Ablösung noch stundenlang die Lebensfähigkeit erhält, zeigt folgender von mir beobachtete Fall:

Georg G., 17 Jahre alt, wurde am 10. April 1917, um 8 Uhr abends, durch ein Au tomobil überfahren und sehr anämisch mit rechter Schlüsselbeinfraktur auf meine Abteilung aufgenommen. Ueber dem linken Schläfenbein ist ein handtellergroßer Hautlappen von der Galea abgelöst. Das linke Ohr hängt an einer dünnen Hautbrücke des Ohrläpp. chens. Er wurde mit einem Deckverband versehen. Injektion von Tetanusantitoxin. Ich $\varepsilon$ ah den Patienten am 11. April, vormittags $10 \mathrm{Uhr}$, alko zehn Stunden nach dem Unfall. Das linke Ohr lag, mit braungrauem Straßenschmutz bedeckt, vollständig abgelöst vorn an der Backe, mit einer dünnen Hautbrücke, nicht dicker wie eine Violinsaite, in Verbindung. Das ganz kühle, livide $\mathrm{Ohr}$ wurde vom Schmutz gesäubert und das $\mathrm{Ohr}$ an die normale Stelle zurück adaptiert. Die hintere Hautbedeckung der Ohrmuschel, die teilweise gequetccht, ganz zurückgezogen war, wurde auf die Knorpeloberfläche der Ohrmuschel angelegt und mit einigen Nähten an die Hautpartie der Vorderfläche fixiert. Dann wurden die einzelnen kleinen Einrisse zwischen Helix und Antihelix mit einzelnen Nähten vereint. Beconderes Gewicht leg te ich darauf, daß das Ohr am äußeren Gehörgang wieder genau adaptiert und vereinigt wєrde, damit das eventuell wieder anwachsende Ohr am Gehörgang keine Striktur oder Stenose zeige.

Der skalpierte Lappen wurde mit einigen Nähten fixiert. Beim Verbandwechsel nach 24 Stunden war das Ohr weniger livid, am dritten Tage nach der Naht wurde die'Farbe der Ohrmuschel normal. Am 15. April Entfernung einiger Nähte zwischen Helix und Antihelix. Nach zwei Wochen an der fest angewachsenen Ohrmuschel zwei kaum linsengroße, granulierende Stellen. Gehör absolut normal.

Im Budapester Aerzte-Verein wurden ähnliche plastische Operationen erwähnt durch Milko, Réthi, Polya. Diese Demonstrationen bezogen sich aber alle auf Fälle, die später operiert wurden als mein mitgeteilter Fall. 